\title{
Les inventions animées, Émile Cohl au prisme d'une histoire culturelle des techniques
}

Animated inventions, Émile Cohl through the prism of a cultural history of technology

\section{Alain Carou}

\section{(2) OpenEdition}

\section{Journals}

Édition électronique

URL : https://journals.openedition.org/1895/2423

DOI : $10.4000 / 1895.2423$

ISBN : 978-2-8218-0997-0

ISSN : 1960-6176

\section{Éditeur}

Association française de recherche sur l'histoire du cinéma (AFRHC)

\section{Édition imprimée}

Date de publication : 1 décembre 2007

Pagination : 140-153

ISBN : 978-2-913758-54-4

ISSN : 0769-0959

\section{Référence électronique}

Alain Carou, "Les inventions animées, Émile Cohl au prisme d'une histoire culturelle des techniques », 1895. Mille huit cent quatre-vingt-quinze [En ligne], 53 | 2007, mis en ligne le 01 décembre 2010, consulté le 17 avril 2022. URL : http://journals.openedition.org/1895/2423 ; DOI : https://doi.org/ 10.4000/1895.2423 


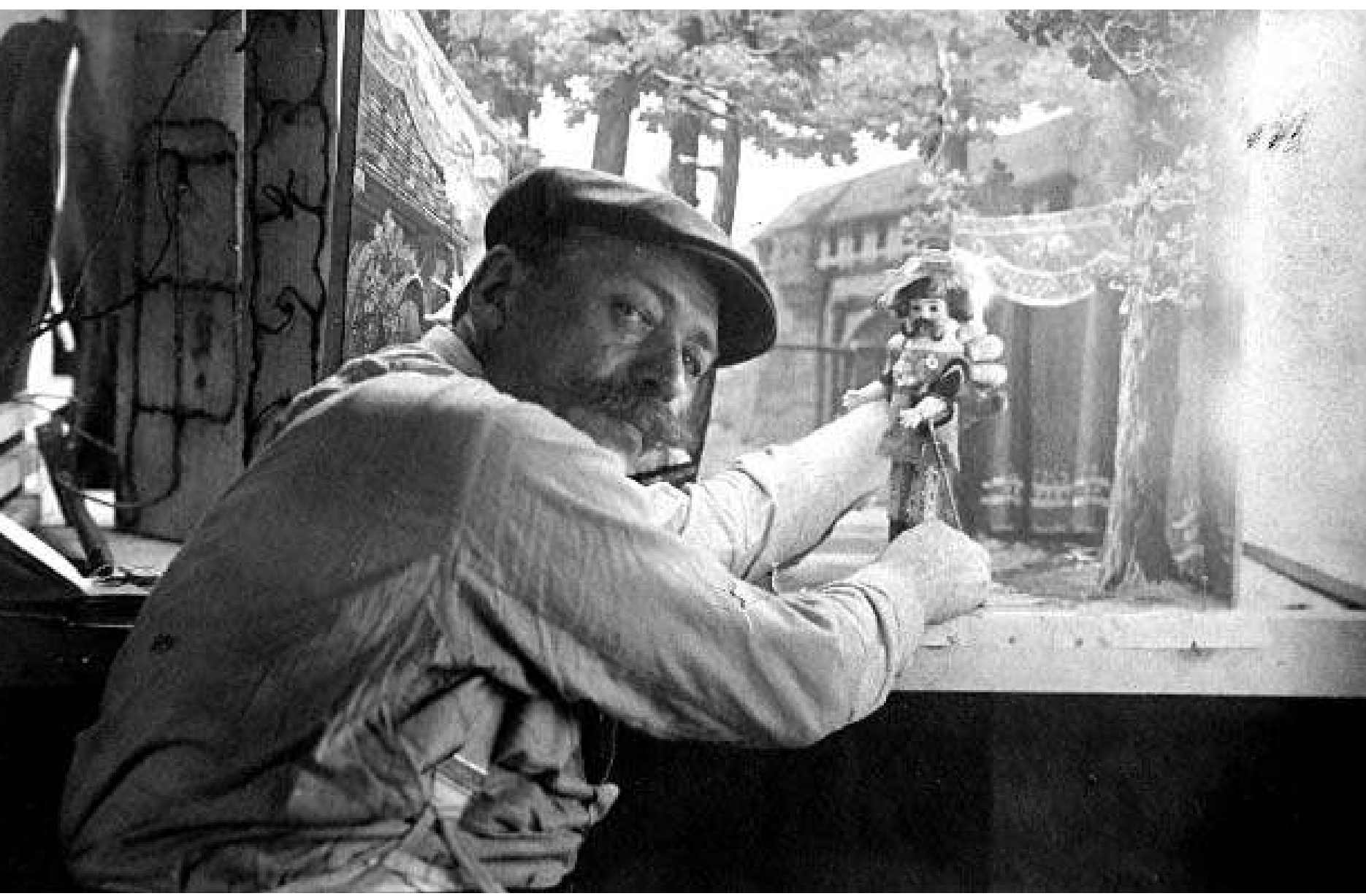

Émile Cohl animant les poupées du Tout Petit Faust (1910) 


\title{
Les inventions animées, Émile Cohl au prisme d'une histoire culturelle des techniques
}

\author{
par Alain Carou
}

Chez Émile Cohl, l'invention graphique s'allie à un goût et à une pratique des techniques à des fins spectaculaires et ludiques. À cet égard, il y aurait sans doute lieu de reprendre le fil d'une histoire "techniciste ", attentive aux outils, aux méthodes et aux savoir-faire de l'animateur à l'œuvre. Toutefois, c'est plutôt dans les termes d'une histoire culturelle des techniques qu'on voudrait s'essayer, dans la présente étude, à approcher les premières activités cinématographiques de Cohl. En d'autres termes, il s'agirait de saisir quelques-uns des jeux de relations existant entre les pratiques techniques d'un homme, considéré comme inventeur, innovateur et exploitant, et les connaissances ou l'imaginaire techniques de son époque.

Cohl entrepreneur de cinéma itinérant, Cohl et les jouets modernes, Cohl et la « découverte " du secret de l'animation : le texte qui suit s'attachera à des sujets en apparence très hétérogènes (biographie, iconographie, historiographie), par ailleurs d'importance et de notoriété très différentes (de la découverte d'une brève activité de Cohl comme " tourneur » en 1907 à la place du réalisateur de Fantasmagorie dans les premières histoires du cinéma). À chaque fois, nous chercherons à appréhender comment les technologies de la représentation et du spectacle s'articulent aux représentations et au spectacle de la technologie, notamment par l'intermédiaire des discours et des dispositifs de promotion de la nouveauté technique. Discours et dispositifs qui obéissent en première instance à la rhétorique de la connaissance et du progrès, mais dont on verra qu'ils ne sont pas dénués d'ambiguïtés. 


\section{Deux exploitants itinérants en quête de spectateurs}

Un fait nouveau, découvert dans les archives personnelles de Cohl, est à l'origine de la présente étude : peu avant de réaliser ses premiers films, I'homme a exploité une micro-entreprise de cinéma itinérant ${ }^{1}$. Dans les carnets où il a reconstitué son existence mois par mois à la fin de sa vie, on lit pour mars 1907 : "Ciné avec Marcel à Orléans - Beaugency Jargeau. Four ${ }^{2}$ ", et pour le mois précédent : "Marcel m'écrit d'Orléans ${ }^{3}$ ». Trois lettres non datées montrent qu'il a été associé dans cette affaire à Marcel Robert, jeune poète et acteur au théâtre d'Orléans ${ }^{4}$. Dans un brouillon de convention, certainement de la main de Cohl, les rôles sont répartis entre les co-associés : à Courtet-Cohl «l'idée et la préparation

$1895 /$

1 II y a cinquante ans, ce fait avait été mentionné par René Brest - qui avait fait campagne pour la reconnaissance de Cohl dans Paris-Soir en 1934 - et André Courtet, le propre fils d'Émile Cohl (« II y a vingt ans, l'inventeur du dessin animé mourait à l'hôpital », les Nouvelles littéraires, 13 février 1958). Ils le situaient approximativement en 1904-1905. Leur affirmation a été ensuite révoquée en doute par Donald Crafton qui n'a pas eu connaissance des documents que nous exploitons ici (Émile Cohl, Caricature, and Film, Princeton, Princeton University Press, 1990, p. 92).

2 Beaugency et Jargeau sont deux petites villes voisines d'Orléans. L'entreprise a-t-elle tourné court très rapidement ou Marcel Robert a-t-il continué l'exploitation quelque temps en l'absence de Cohl ? Les archives ou la presse locales permettraient peut-être d'en savoir plus sur ce point.

3 En fait, le début du projet pourrait remonter à la fin de 1906, étant donné la proposition faite par Marcel Robert d'organiser une première séance à destination des enfants d'Orléans, avec distribution de jouets, un 24 (décembre très probablement).

4 II s'agit du futur réalisateur Robert Péguy. Son état-civil exact semble être Marcel Robert Péguy (18831968), mais il signera ses scénarios Marcel Robert quand Cohl I'introduira chez Gaumont. Sur la foi d'une interview mal retranscrite (dans I'Image, $n^{\circ} 36,1932$ ) - qui passe par ailleurs complètement sous silence l'épisode de l'exploitation itinérante - il a été maintes fois qualifié de gendre d'Émile Cohl. Mais Robert Péguy n'a jamais été le gendre d'Émile Cohl. Il était le fils, issu d'un mariage précédent, de la troisième épouse du peintre Hyppolite-Camille Delpy. H.-C. Delpy fut veuf deux fois et se maria trois fois. Sa fille Suzanne, épouse d'Émile Cohl, était issue de son premier mariage. La fille de Robert Péguy, Micheline, était la filleule d'André Courtet, fils d'Émile Cohl et de Suzanne (informations aimablement fournies par Pierre Courtet-Cohl). 
équilibrée des durées). C'est également Cohl qui rédige un mode d'emploi rappelant les précautions à prendre lors de l'éthérisation du projecteur et qui est interrogé par son parent sur les risques inhérents au gaz utilisé lors de sa première demande de location de salle à Orléans.

Aux 39 minutes de projection cinématographique s'ajoutent plusieurs attractions, l'ensemble devant approcher deux heures au total selon le calcul de Marcel Robert. Cohl a fait l'acquisition d'un chromatrope - un appareil optique permettant la projection de rosaces colorées en rotation, effet obtenu en faisant tourner à rebours l'un de l'autre deux disques de verre juxtaposés, peints de manière semblable. On obtient ainsi des effets de type kaléidoscopiques, avec des formes géométriques lancées dans toutes les directions ${ }^{5}$. Bien qu'il n'y ait pas trace de projections fixes dans le projet, le chromatrope leur est souvent combiné, de manière à obtenir des effets de neige qui tombe, d'eau en écoulement, de fumées, etc. ${ }^{6}$

De son côté, Marcel Robert doit jouer des monologues sous son nom de scène : « Dubreuil, du théâtre d'Orléans ${ }^{7}$ ". Les deux associés disposent aussi d'un phonographe dernier cri " Homophone " (marque lancée en Allemagne en 1905 et déposée en France en 1907), acheté à la maison Girard, principal revendeur de phonographes sur la place de Paris. Cet appareil était-il principalement destiné à l'accompagnement des projections ? Bien qu'aucun frais lié à l'accompagnement par un musicien ne soit indiqué dans les calculs de prix de revient, cela n'est pas certain. D'une part, Marcel Robert mentionne explicitement à Cohl qu'il « compte utiliser l'Homophone pour le défilé des soldats », ce qui fait sans doute référence à la vue de 110 mètres intitulée en abrégé " Armée » dans le programme. Or il n'aurait pas lieu d'écrire cette phrase si l'accompagnement des films au phono était la règle. D'autre part, Marcel Robert veut demander à la maison Girard des disques « de morceaux d'orchestre ou de monologues de Dranem, Polin, etc. " : cette seconde catégorie d'enregistrements est incontestablement destinée à une audition séparée, or la conjonction semble indiquer qu'elle est interchangeable avec le genre pièces orchestrales - donc que celles-ci sont aussi destinées à être jouées hors projections. Calculant la durée du spectacle, Marcel Robert écrit bien d'ailleurs que « la sauce est allongée par l'Homophone ».

En ce qui concerne le « standing » de leur programme, les deux acolytes semblent d'accord : cette "petite entreprise artistico-cinématographique " vise au départ un "élégant

5 « Chromatropes », le Magasin pittoresque, 1878, pp. 79-80.

6 Notons au passage que Cohl emploiera des effets de chromatrope dans au moins un de ses films, Cadres fleuris (1910).

7 L'identification de Marcel Robert au « M. Dubreuil » annoncé dans le programme est permise par le fait que la tournée s'appelle « Dubreuil et compagnie " et qu'aucun frais d'acteur n'est prévu dans les calculs de prix de revient. Par ailleurs, Marcel Robert escompte des facilités (imprimerie, salle) grâce à ses relations avec Marcel Dupuis, directeur du théâtre d'Orléans entre 1907 et 1914. 
public ». Les monologues sur disque, la mention "Dubreuil, du théâtre d'Orléans " sont destinés à «fai[re] bien ». Marcel Robert propose à Cohl d'accompagner en été le déplacement du théâtre d'Orléans à Pougues-les-Eaux, villégiature thermale. Dans l'immédiat, il projette d'organiser une séance spéciale à l'Institut d'Orléans (probablement pour la Noël 1906) avec distribution de jouets aux enfants : les tarifs d'entrée qu'il prévoit (1 F. et 0,75 F.) sont élevés. Quant à Cohl, il veut au printemps « remonter le littoral au moment où les Parisiens arrivent ".

Cependant, les deux associés songent également aux publics locaux des petites villes et villages, qu'ils comptent traverser au cours de leur itinéraire en direction de la mer (Vendôme, La Flèche, Morée Saint-Hilaire, Segré...). Ils prévoient de se déplacer par le train avec la cargaison de matériel, puis, une fois arrivés, de solliciter un emplacement à la mairie et d'assurer la réclame.

L'argumentaire met l'accent sur le caractère convenable du spectacle (on peut y amener ses enfants) :

Nous avons l'honneur de vous présenter un spectacle dit « de famille ». Nulle inconvenance, soirée récréative au possible composée de vues cinématographiques d'un comique abracadabrant, forçant les rires les plus rebelles et bien faite pour guérir toute personne atteinte de neurasthénie, hypocondrie et autres maladies imaginaires de ce genre. Citons au hasard quelques titres de vues: l'Enfant coupé en deux; la Pêche miraculeuse; le Voleur, le Policeman et le Balayeur (scène anglaise); I'Incendie de tout un quartier d'une ville américaine, avec écroulement de maisons ; les Petits Coupeurs de bois vert (scène fort attendrissante) ; I'Assassinat du courrier de Lyon, vue en 4 tableaux : I'attaque, le rapt, les voyageurs prisonniers, délivrés !!

Si ce discours emprunte largement aux formes du boniment oral, c'est par le prospectus, médium de l'écrit, que Marcel Robert juge habile de le propager. En effet, il estime que dans les villages, « le tambour ne sera pas utile, même inutile, si nous l'employons seul, sans prosp[ectus]. Je connais les bons indigènes de nos campagnes. Il suffit qu'après avoir tambouriné le tambour commence "Ce soir grrrande repré..." pour qu'aussitôt les portes et les oreilles se ferment. "Des blagues" disent les braves gens, et le tour est joué. Pas un sou de recette ». Dans les villes comme Vendôme, " il n'y a plus de tambour et ce ne sont pas 200 prospectus que nous distribuerons dans cette ville qui nous attireront beaucoup de monde " : on devra donc compter là sur le placardage de "grandes affiches qui comprendront le programme détaillé ». 
Jusqu'à ce point, des conceptions relativement « modernes " président à l'entreprise : celles d'un public plus homogène qu'avant entre ville et campagne, réceptif à la propagande écrite et méfiant à l'égard du boniment. Marcel Robert aborde pourtant tout différemment la promotion des techniques dans le prospectus destiné aux «trous pas chers ». II conseille en effet à Cohl de couper dans ses explications détaillées car « vouloir trop faire comprendre au public est une faute souvent et [...] toujours un peu de curiosité préside au désir de se rendre à l'appel des troupes de passage » : le cinématographe et le phonographe feront une meilleure attraction nimbés d'un peu de mystère quant à leurs principes de fonctionnement. Quant à l'effet qu'ils produisent, leur nom doit l'exprimer de façon limpide. Marcel Robert et Émile Cohl forgent ainsi de toutes pièces des dénominations parlantes : leur projecteur sera « le Merveilleux Cinémato-Universel » (préféré au « Cinématographe Soleil »), et l'Homophone un « Phono-Chantographe, machine parlante perfectionnée 8 "; le "Chromatrope " conserve son nom mais devient " éblouissant " - un nom encore " bien difficile à digérer pour nos paysans ", commente tout de même Marcel Robert. Dans ce discours, les technologies d'enregistrement et de reproduction rejoignent la panoplie de l'illusionnisme.

\section{Jouets, mouvement et illusion}

C'est de nouveau en 1907 que se situe le point de départ de notre second axe d'étude. Cette année-là, Émile Cohl participe au concours-exposition des inventeurs de jouets, institué à Paris par le préfet Lépine (cet événement ne deviendra que plus tard le concours d'inventeurs en tous genres que l'on connaît sous le nom de « Concours Lépine »). Parmi les treize inventions distinguées par la Nature cette année-là, se trouve son $A B C D$ à la ficelle ${ }^{9}$. C'est " un petit casse-tête très instructif destiné à graver la forme des lettres dans l'esprit des enfants et à exercer leur sagacité. On met à leur disposition un alphabet de lettres majuscules comme modèles, un cordon, une planchette sur laquelle sont plantés une quarantaine de clous. La disposition de ceux-ci est telle qu'en faisant passer le cordon autour de certains d'entre eux on peut reproduire toutes les lettres et les chiffres ". L'ABCD à la ficelle fait le lien entre le concepteur inlassable de jeux instructifs et casse-tête pour la presse et le futur réalisateur de dessins animés. D'un ensemble de points dispersés, l'enfant est appelé à tirer une succession de formes identifiables : on retrouvera le même principe figuratif (mais dénué de fonction pédagogique) dans des dessins animés comme les Joyeux

8 Rappelons que le terme de "machine parlante » est d'usage commun à cette époque.

9 La Nature, supplément $2^{\mathrm{e}}$ semestre 1907, p. 156. 


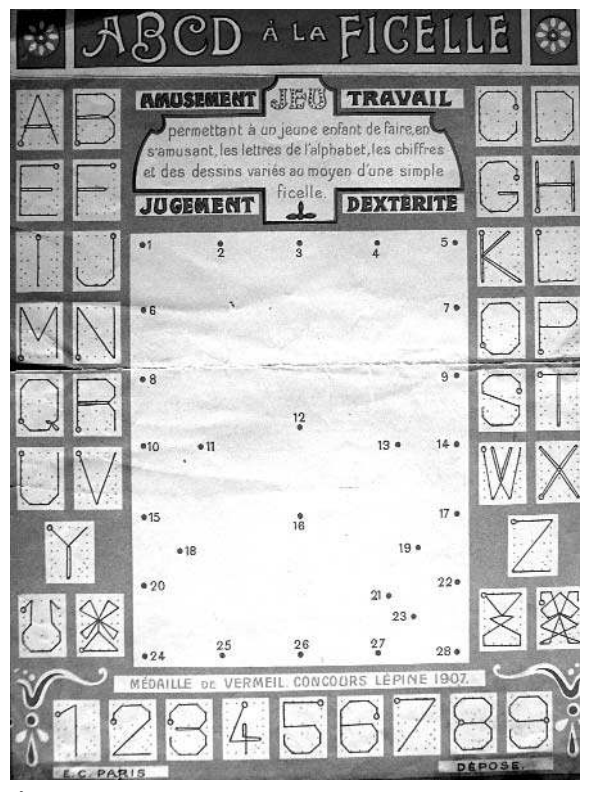

Émile Cohl, $A B C D$ à la ficelle, 1907
Microbes, où un nuage de points dispersés s'ordonne et se réordonne en une involution de figures reconnaissables.

Toutefois, on voudrait plutôt s'attarder ici sur le fait même que Cohl ait participé au concours institué par Lépine. Car le jeu et le jouet sont fréquemment présents dans ses films, toujours en mouvement : bilboquet dans Fantasmagorie, cartes, jeu de volant et soldats de plomb dans Affaires de cœur, poupée de chiffon dansant dans les Locataires d'à côté, pantin à fils et figurines enfantines dans les Lunettes féeriques, ourson de peluche de Rêves enfantins, pantins articulés à plat ou en volume dans Soyons donc sportifs - voire le puzzle du Champion du jeu à la mode.

Commençons par la part d'explication biographique : dans les carnets où il a reconstitué la chronologie de sa vie, Cohl mentionne en 1905 et 1906 les jouets commandés pour Noël par son jeune fils André (né en 1899), sur la foi de lettres que selon toute vraisemblance il a conservées. On peut imaginer que Cohl s'est intéressé tardivement au jouet par affection paternelle. Difficile d'aller plus loin dans cette hypothèse qui touche à son intimité.

Mais le contexte culturel invite à élargir la perspective. Car autour du jouet se nouent de façon caractéristique, en ce début de siècle, le divertissement enfantin, la vulgarisation technique sur fond de compétition économique internationale et l'esprit amateur cultivé. Au tournant du siècle, les Allemands ont conquis la première place sur le marché du jouet en Europe, en employant des matériaux industriels bon marché et en massifiant la production. Cette réussite est illustrée de façon emblématique par le Teddybär, lancé en 1902 par Margaretha Steiff. Comme face à toute manifestation du dynamisme allemand, la France s'inquiète. En témoignent entre autres le rapport détaillé de la section jouets à l'Exposition universelle de 1900 et celui de la World's Fair de 1904 à Saint-Louis (États-Unis). Leurs rédacteurs vantent la tradition parisienne du jouet et ses qualités supposées toutes françaises de raffinement et d'inventivité, mais pour mieux l'encourager à trouver un second souffle et 
à s'organiser pour affronter les enjeux du monde contemporain. Le concours institué par Lépine en 1901 vise précisément à encourager l'innovation en matière de jouets à bas prix.

Cependant, l'aspiration à une renaissance du jouet national est indissociable de l'essor d'un esprit de connaissance érudite et de collection, comme l'atteste la proximité entre la Société des amateurs de jouets anciens (fondée en 1902) et la Chambre syndicale du jouet. Industrialisme et nostalgie sont les deux pans solidaires d'un discours porté par les mêmes personnes. Savants historiens et collectionneurs, mais aussi observateurs minutieux des nouveautés et rédacteurs des rapports susmentionnés, deux hommes incarnent au mieux cette convergence d'intérêts : Léo Claretie - par ailleurs romancier pour enfants et vulgarisateur de la culture du xvIII siècle - et surtout Henry-René d'Allemagne. Érudit hors normes, celui-ci a soutenu à l'École des chartes une thèse sur la serrurerie au Moyen Âge (avec démonstration pratique en soutenance !). Vivant de sa fortune personnelle, il exerce bénévolement les fonctions de bibliothécaire surnuméraire à l'Arsenal. Dans sa bibliographie pléthorique, dominée par l'histoire des techniques, des jeux et du mouvement saintsimonien, il est périlleux de cerner une unité. Elle résiderait peut-être dans un intérêt multiforme pour la culture matérielle et l'histoire de l'innovation ${ }^{10}$.

Autre aspect important, l'industrie du jouet des années 1900 poursuit l'illusion de la vie et du mouvement appliquée à une production de masse. À la suite d'Émile Jumeau, inventeur des visages de poupées à caractère expressif (le "bébé Jumeau "), la Société française de fabrication de bébés et jouets récupère son travail et domine jusqu'aux années trente le marché des jouets à prix modique. Entre 1903 et 1910, elle dépose des brevets pour bébé parleur, poupée mécanique parlant et gesticulant combinable avec une voiture, animal-jouet marcheur, bébé à yeux mobiles, bébé fumeur ${ }^{11}$. De son côté, d'Allemagne admire les vitrines derrière lesquelles des jouets animés miment la société à échelle réduite : «M. Fernand Martin [...] avait représenté avec ses amusantes petites poupées automatiques une fête de village avec chevaux de bois et divertissements variés. Tout le monde de la rue, le marchand d'oranges, le balayeur, les acrobates, etc., sans oublier le représentant de l'autorité, fraternisait ensemble avec une entente vraiment touchante12. " La Nature qualifie de "petites merveilles d'ingéniosité et de vérité de mouvement " les réalisations du même Martin,

10 Sur Henry-René d'Allemagne, voir Revue de la Bibliothèque nationale de France, $\mathrm{n}^{\circ} 21, \mathrm{pp} .67-69$. 11 Anne-Marie Porot, Jacques Porot, François Theimer, Histoire et étude de la Société française de fabrication de bébés et jouets, Paris, Éditions de I'Amateur, 1994.

12 Henry-René d'Allemagne, les Jouets à la World's Fair..., Exposition internationale de Saint-Louis. Section française, rapport du groupe 36, Paris, 1907, p. 20. 
instantanés de la vie de la rue : le pompier montant à l'échelle, le joueur de boules, l'éplucheur de légumes, la voiture à asphalte ${ }^{13}$. Un petit monde autonome s'anime sous le regard de l'enfant devenu spectateur des jouets. Pour la Noël 1909, le fabricant Roullet-Descamps met en place au Bon Marché la première vitrine de jouets animés à Paris, participant d'une débauche d'effets visuels destinés à entretenir le désir de consommation ${ }^{14}$.

Regard rétrospectif sur le jouet traditionnel, transformation du jouet moderne en spectacle vivant offert à distance : ces données, liées à l'avènement d'un âge technologique du loisir enfantin, traversent une partie de l'œuvre cinématographique de Cohl. Dans le Petit Soldat qui devient dieu, les figurines bon marché de deux enfants s'animent et jouent la vie de la mation propre au jouet moderne.

Dans Soyons donc sportifs, des figurines de bois ou de carton reproduisent plaisamment tout le répertoire des loisirs de mouvement, et notamment des plus en vogue : automobile, patin, bicyclette, barque... Là où les vitrines d'automates figurent une société humaine où tout «fonctionne ", les petits automates de Cohl terminent à chaque fois leur parcours par un accident, une chute, une panne.

De manière exemplaire, Monsieur Clown au pays des Lilliputiens figure la transformation du jouet en spectacle sophistiqué autonome que l'on " touche seulement avec les yeux ". Sur une piste de cirque, devant un public animé d'expressions diverses, clairement séparé, le clown, l'éléphant, l'écuyère se livrent à des acrobaties. Celles-ci s'apparentent à des enchaînements de mouvements mécaniques que les jouets animés réels effectuent seulement par portions et en boucle. Les couples de jouets liés, se livrant simultanément à des mouvements distincts, tels le cheval et l'écuyère, sont caractéristiques de la nouvelle offre ludique.

Plus tardifs, Chantecler et le Tout Petit Faust sont dépourvus de l'ambiguïté propre au dispositif (ou au retournement de dispositif) des films précédemment cités. Les jouets réalisent là une véritable petite représentation, réalisant l'horizon ultime de la vitrine de jouets animés (l'ironie ne se perçoit plus guère que dans la forme raccourcie à l'extrême des pièces d'origine). Cohl

13 "Quelques jouets nouveaux », la Nature, 3 décembre 1904, p. 64.

14 Michael B. Miller, The Bon Marché. Bourgeois Culture and the Department Store, 1869-1920, Princeton, Princeton University Press, 1981, p. 169. 


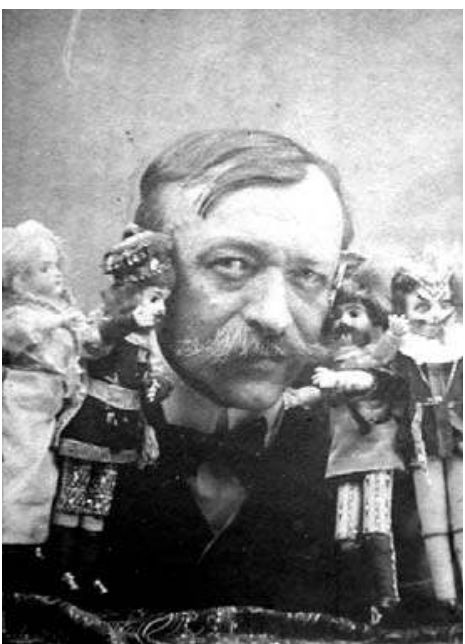

Émile Cohl

et les poupées du Tout Petit Faust (1910)

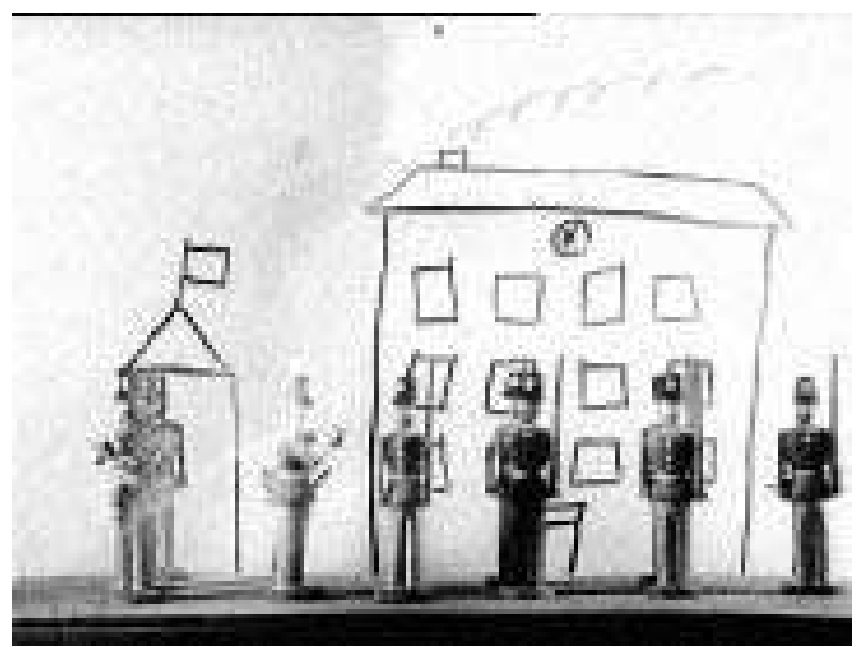

Émile Cohl, le Petit Soldat qui devient dieu (1908) emploie un type de jouets d'origine généralement allemande qui, selon d'Allemagne, remportent un vif succès en France à cette époque. La simplicité délibérée et la modicité des jouets employés dans les films précédents cèdent le pas à des productions plus luxueuses. Margaretha Steiff, entre autres, s'est fait une spécialité de ces animaux de feutre et d'étoupe sur armature métallique. «Les types les plus fréquents sont les chiens, les chats, les cochons, les ours et les singes. La solidité de ces articles est vraiment très remarquable. [...] Parmi les modèles présentés par Mme Steiff, nous signalerons entre autres quelques jolis oiseaux tels que des faisans, des coqs de basse-cour exécutés à l'aide de morceaux de feutre découpés qui produisent un fort joli effet ${ }^{15}$. " Comme la basse-cour forme justement le décor de Chantecler, il y avait une coïncidence propre à intéresser Cohl. De même le choix du Tout Petit Faust n'est-il probablement pas étranger à l'ancrage nurembourgeois de l'industrie du jouet en Allemagne.

Fait révélateur : en 1937, à l'époque où l'invention des primitifs du cinéma bat son plein en France, Henry d'Allemagne est nommé rapporteur pour la remise d'une récompense à Émile Cohl, « inventeur du dessin animé », de la part de la Société d'encouragement pour l'industrie nationale ${ }^{16}$.

15 Henry-René d'Allemagne, les Jouets à la World's Fair..., op. cit., pp. 43-44.

16 Bulletin de la Société d'encouragement pour l'industrie nationale, pp. 113-116. À noter que d'Allemagne attribue à Cohl la paternité de la bande le Travail se fait tout seul, probablement sur la base de l'attribution erronée de ce film Vitagraph (Work Made Easy) à Gaumont dans I'Illustration en 1908 (cf. infra). Sur l'invention des primitifs, voir Christophe Gauthier, " L'invention des "primitifs" à l'orée du parlant : le cas Méliès ", Cahiers parisiens, vol. 2, 2006, pp. 148-174 ; et sa thèse Une composition française. Histoire de la mémoire du cinéma en France des origines à la Seconde Guerre mondiale, Paris I, 2006. 


\section{L'animation cinématographique ré-inventée}

En apparence, la soi-disant découverte par Cohl du secret de l'animation constitue une curieuse anomalie dans la première historiographie du cinéma. Dans le Cinéma pour tous (1922), Étienne Arnaud et Boisyvon écrivent que l'Hôtel hanté de la Vitagraph intrigua beaucoup les éditeurs français en 1907, et que ce fut Cohl qui sut découvrir le principe de l'animation des objets par la prise de vue image par image. Donald Crafton a fait un sort à cette légende en montrant que des metteurs en scène français avaient déjà recouru à ce procédé - ainsi Gaston Velle et Segundo de Chomòn avec le Théâtre du petit Bob édité par Pathé17 - et que les techniciens de Gaumont ne pouvaient l'ignorer. Comment expliquer alors ce récit des origines ? Dans son étude extrêmement serrée, Crafton avance entre autres que la thèse d'une « découverte » soudaine permettait de justifier le fait que l'industrie française n'ait investi dans l'animation que sous la pression de la concurrence de la Vitagraph ${ }^{18}$.

17 Notons au passage que cette bande très soignée représente déjà des jouets doués de vie. Ils se donnent en spectacle sous le regard d'acteurs enfantins réels, répétant littéralement le dispositif de la vitrine de jouets animés - ce qui ne se retrouve pas dans les films d'Émile Cohl.

18 Donald Crafton, op. cit., pp. 121-138.

19 « Il est évident qu'une ambiance de secret fut créée délibérément autour de la technique de l'animation. [...] [Cela] peut être vu comme la marque de producteurs désireux [...] de faire passer la technique et les effets pour uniques et de grande valeur - à l'instar de la magie - plutôt que pour la simple opération mécanique en quoi ils consistent en réalité. » (P. 137, la traduction est de nous.) 
II y a une hypothèse complémentaire (au demeurant également esquissée aussi par Crafton ${ }^{19}$ ) : elle consiste à réinscrire les débuts cinématographiques de Cohl dans une mutation importante du regard porté sur les "trucs". L'Illustration, revue à grande diffusion, publie au printemps 1908 un article bien documenté sur ce sujet20. Gaumont a fourni l'information et les illustrations, et s'attire les foudres d'une partie de la profession. Pour Georges Méliès, un truc expliqué perd sa valeur :

Je trouve que la divulgation des procédés secrets de fabrication à un public de profanes est la plus lourde faute qu'il soit possible de commettre : vous n'ignorez pas que, étant depuis de longues années directeur du théâtre Robert-Houdin, je suis habitué de longue date à inventer et à construire des " trucs»» de toutes sortes; qui tout en reposant sur des principes connus, semblent mystérieux à ceux qui ne savent pas démêler ces principes. [...] Le truc luimême n'est pas chose surnaturelle, toute la valeur est dans l'adresse avec laquelle le moyen employé est caché aux yeux du public [...] il est positivement absurde d'aller expliquer ce procédé à tout venant [...] vous détruisez vous-mêmes le fruit de votre travail puisque vous anéantissez l'illusion [...] le syndicat des Illusionnistes de France, dont je suis président, a d'ailleurs dans ses statuts un article interdisant à ses membres toute divulgation des secrets de la prestidigitation ${ }^{21}$.

Méliès défend une représentation artisanale du film à trucs : ses bandes jouent d'une combinaison de secrets de métier d'une manière à chaque fois particulière. À l'opposé, I'Illustration délivre des explications techniques générales illustrées par des exemples notamment le "procédé des arrêts " avec l'exemple du Bon Écraseur - et construit des typologies. Même enrobée dans un style journalistique léger, cette opération de vulgarisation constitue à proprement parler un discours technologique. Or celui-ci marque une rupture avec l'esprit des attractions. L'article de l'Illustration affirme en effet que les trucs ne valent que s'ils permettent " des effets dramatiques nouveaux ". La puissance de disruption que les apparitions-disparitions par arrêt caméra possèdent dans le cinéma des attractions laisse place à un nouveau modèle où elles sont les points de focalisation d'une progression inscrite dans la durée.

20 Cf. Roland Cosandey, "Cinéma 1908, films à trucs et Film d'Art : une campagne de I'lllustration ", Cinémathèque, $\mathrm{n}^{\circ} 3$, 1993, pp. 58-71. André Gaudreault et Jean-Pierre Sirois-Trahan ont retrouvé l'iconographie de ce numéro largement reprise dans le journal québécois la Patrie du 31 juillet 1909, témoignant de son écho considérable (la Vie ou du moins ses apparences, Montréal, Cinémathèque québécoise/Grafics, 2002, p. 80).

21 Georges Méliès, " Les coulisses de la cinématographie ", Phono-Cinéma-Revue, n² 2, avril 1908. 
Comme on l'a dit, I'lllustration s'attarde sur l'exemple de la bande comique le Bon Écraseur : une automobile écrase un pochard et lui laisse les deux jambes sectionnées ; le propriétaire de la voiture sort ses outils et répare l'homme coupé en deux. Une portion de la bande reproduite détaille les phases successives de la séquence qui voit le véhicule s'approcher dangereusement du pochard. On peut se demander si ce type d'image, fixant l'expérience fuyante de la projection, ne contribue pas à un changement de la perception de la temporalité du cinéma. L'événement lié au truc n'est plus perçu comme étant d'ordre instantané, mais amené dans la durée. Maint lecteur, devant cette bande de photogrammes, peut se livrer même pour la première fois (ou presque) à une opération imaginaire de renversement ou d'étirement du temps. Le philosophe Alain paraît traduire assez bien ce changement de perception quand il écrit en 1910, à propos de la différence entre une mort accidentelle imaginée et une mort accidentelle vécue : « Aussi, moi qui pense à l'accident, j'en juge très mal. J'en juge comme un homme qui, toujours sur le point d'être écrasé, ne le serait jamais. [...] Je me donne une vue cinématographique de mon propre écrasement, mais une vue ralentie, et même arrêtée de temps en temps; et je recommence ; je meurs mille fois et tout vivant22. " La coïncidence est assez frappante pour qu'on soit en droit de supposer qu'Alain gardait quelque souvenir de la lecture de l'Illustration.

Le discours technologique a part liée avec l'investissement des trucs dans la narration cinématographique. Le spectateur va de plus en plus adresser au film à « trucages » un regard dédoublé : d'une part en occupant une position intégrée au récit cinématographique, d'autre part en portant un regard distancié informé (ou s'efforçant de l'être) sur les moyens employés. Les trucs quittent la panoplie de l'illusionniste pour entrer dans le domaine réglé des technologies. Nul hasard à ce que Gaumont soit en France le catalyseur de cette évolution du régime des trucs. II est le principal propagateur d'une appréhension du cinéma par le prisme technologique dans ces années, assurant la promotion de ses innovations (projecteurs, Chronophone, trichromie) par les canaux de la presse de vulgarisation. II présidera aussi pour une bonne part à la constitution de la première historiographie « techniciste » officielle dans les années vingt ${ }^{23}$.

Dans ce démantèlement de l'illusionnisme comme mode de réception, le secret est gardé sur une seule espèce de "truc » : le procédé d'animation image par image. Sous la plume du rédacteur de I'Illustration, l'opérateur Anatole de Thiberville revendique la découverte

22 Alain, "Maux d'esprit », la Dépêche de Rouen, 12 décembre 1910 ; repris dans Propos sur le bonheur, 1928, rééd. Paris, Gallimard, « Folio », 1985, p. 30.

23 Christophe Gauthier, op. cit. 
du « secret » de la Vitagraph, mais en reporte à plus tard la révélation au public : " Je viens de réussir la première de ce genre. Mais quant à vous en dévoiler le mystère [...] c'est tout à fait impossible, quant à présent. "Et, reprend Gustave Babin, " en vérité je suis persuadé qu'il faut que ce soit un gros secret pour qu'on se défende de me le divulguer ». Le secret est en réalité prédestiné à une courte vie, puisque Babin incite les lecteurs à en imaginer la solution, suggérant qu'elle est imaginable; et que Lectures pour tous finit par vendre la mèche en juin 1908, dans un article qui porte aussi l'empreinte de la maison Gaumont. Par cet empressement à attirer l'attention sur un procédé en l'environnant de mystère, puis à le dissiper rapidement, l'animation image par image remplit le rôle de la technique qui par elle-même fait attraction. Dans une appréhension désormais structurée des trucs, une dichotomie très nette se dessine, entre des trucs "traditionnels ", désormais annexés au cinéma dramatique, et un procédé "nouveau », constituant par lui-même une nouvelle attraction, vouée pour ses premières apparitions à déployer ses effets pour eux-mêmes et pour l'étonnement du spectateur. Redistribution décisive pour le statut historiographique des films empruntant à cette technique qui sortent alors. Parce qu'ils sont immédiatement consécutifs à cet avènement de l'animation comme technique à part, les premiers films réalisés par Cohl marquent, sur le plan des représentations sociales (et seulement celui-là), une invention de l'animation. 